\title{
Is there tonic immobility in humans? Biological evidence from victims of traumatic stress
}

\author{
Eliane Volchan ${ }^{\mathrm{a}, *}$, Gabriela G. Souza ${ }^{\mathrm{b}}$, Camila M. Franklin ${ }^{\mathrm{a}}$, Carlos E. Norte ${ }^{\mathrm{a}}$, Vanessa Rocha-Rego $^{\mathrm{a}}$, \\ Jose M. Oliveira ${ }^{a}$, Isabel A. David ${ }^{c}$, Mauro V. Mendlowicz ${ }^{c}$, Evandro Silva Freire Coutinho ${ }^{\mathrm{d}}$, \\ Adriana Fiszman ${ }^{\mathrm{e}}$, William Berger ${ }^{\mathrm{e}}$, Carla Marques-Portella ${ }^{\mathrm{e}}$, Ivan Figueira ${ }^{\mathrm{e}}$ \\ a Instituto de Biofísica Carlos Chagas Filho, Universidade Federal do Rio de Janeiro, Rio de Janeiro, Brazil \\ ${ }^{b}$ Departamento de Ciências Biológicas, Universidade Federal de Ouro Preto, Ouro Preto, Brazil \\ ${ }^{\mathrm{c}}$ Universidade Federal Fluminense, Niterói, Brazil \\ d Escola Nacional de Saúde Publica, Fundação Oswaldo Cruz, Rio de Janeiro, Brazil \\ e Instituto de Psiquiatria, Universidade Federal do Rio de Janeiro, Rio de Janeiro, Brazil
}

\section{A R T I C L E I N F O}

\section{Article history:}

Received 11 November 2010

Accepted 6 June 2011

Available online 5 July 2011

\section{Keywords:}

Tonic immobility

PTSD

Script-driven

Posturography

Heart rate

Heart rate variability

\begin{abstract}
A B S T R A C T
Tonic immobility, characterized by profound motor inhibition, is elicited under inescapable threat in many species. To fully support the existence of tonic immobility in humans, our aim was to elicit this reaction in a laboratory setting and measure it objectively. To mimic exposure to life-threatening events in the lab, trauma-exposed participants with PTSD $(n=18)$ and without PTSD $(n=15)$ listened to the script of their autobiographical trauma. Posturography and electrocardiography were employed. Reports of script-induced immobility were associated with restricted area of body sway and were correlated with accelerated heart rate and diminished heart rate variability, implying that tonic immobility is preserved in humans as an involuntary defensive strategy. Immobility reports seemed more evident in PTSD, suggesting that, in some patients, tonic immobility may be elicited during re-experiencing episodes in daily life. This study provided a measure of tonic immobility, a peritraumatic reaction for which cumulative clinical evidence had linked to the severity of PTSD.
\end{abstract}

(ㄷ) 2011 Elsevier B.V. All rights reserved.

\section{Introduction}

Tonic immobility has been studied in animals for over three centuries (Maser and Gallup, 1977). It is elicited in a context of inescapable threat and is characterized by reversible, profound state of physical inactivity and relative unresponsiveness to external stimuli. Studies in rodents on the neural underpinnings of tonic immobility revealed an intricate set of connections involving several neurotransmitters and brain structures including the periaqueductal gray matter (Monassi and Menescal-De-Oliveira, 2004; Vieira et al., 2011), hypothalamus (Oliveira et al., 1997), amygdala (Leite-Panissi et al., 2003; Leite-Panissi et al., 2006), raphe nucleus (Ferreira and Menescal-De-Oliveira, 2009), and anterior cingulate (Coutinho and Menescal-De-Oliveira, 2010) in a neural network of great complexity. Tonic immobility is considered the last-ditch defense against entrapment by a predator within a sequence of defensive responses: freeze, flight, fight and tonic

\footnotetext{
* Corresponding author at: Institute of Biophysics Carlos Chagas Filho, Federal University of Rio de Janeiro, Av. Carlos Chagas Filho 373, Rio de Janeiro 21941-902, Brazil. Tel.: +55 21 25626556; fax: +55 2122808193 .

E-mail addresses: evolchan@biof.ufrj.br, evolchan@pq.cnpq.br (E. Volchan).
}

immobility (Ratner, 1967). The initial stage of freezing, also called "attentive immobility" (Marks, 1987), is a common adaptive defensive behavior when a potential threat is detected. Being motionless increases the chances to go unnoticed by a predator. If attack starts, overt defense (flight or fight) takes place. When life-threat escalates in the confrontation with a predator, tonic immobility occurs, reducing the probability of continued attack. Different from the attentive immobility (i.e. freezing), which occurs in the lowest threat stage of the defense cascade, tonic immobility is a last antipredator resort when survival is extremely threatened.

Some researchers have studied human psychophysiology in the perspective of this defense cascade model (Bradley et al., 2001; Lang et al., 1997; Mobbs et al., 2009), but they focused on the freeze, flight, and fight reactions, overlooking tonic immobility.

Traumatic events involving intense and inescapable life threats may have the power to evoke tonic immobility-like reactions in humans. Indeed, reports of immobility in life-threatening events from female victims ("rape paralysis") may parallel tonic immobility in other species (Galliano et al., 1993). Recent studies assessed this phenomenon through retrospective reports of the traumatic events in a more systematic way using psychometric instruments in female victims of sexual assault (Bovin et al., 2008; Fuse et al., 2007; Heidt et al., 2005; Humphreys et al., 2010), female and male 
Table 1

Sample characteristics.

\begin{tabular}{lcc}
\hline & With PTSD & Without PTSD \\
\hline Gender & 9 & 9 \\
Male & 9 & 6 \\
Female & & 5 \\
Marital status & 5 & 8 \\
$\quad$ Single & 11 & 2 \\
Married/stable union & 2 & $41.9( \pm 9.01)$ \\
Divorced/widowed & $40.4( \pm 8.03)$ & \\
Age & & \\
\hline
\end{tabular}

students exposed to different types of trauma (Abrams et al., 2009; Bados et al., 2008) and mixed-gender patients with posttraumatic stress disorder (PTSD) secondary to urban violence (Fiszman et al., 2008; Lima et al., 2010; Rocha-Rego et al., 2009). The latter studies in violence victims revealed that the occurrence of tonic immobility during the traumatic event correlates with the severity of PTSD symptoms and refractoriness to pharmacological treatment.

To fully support the existence of tonic immobility in humans, studies on its biological correlates are necessary. In contrast to animal models, tonic immobility in humans has never been studied in the laboratory. The most salient feature of the "tonic immobility" construct is, as its label implies, the motor attribute. Posturographic recording in humans has provided significant insights into immobility during the freezing stage of the defense cascade (Azevedo et al., 2005; Facchinetti et al., 2006), indicating that it might be a viable approach to study tonic immobility in the laboratory.

A paradigm to investigate human tonic immobility in controlled conditions should mimic exposure to life-threatening events. We studied trauma-exposed victims with and without PTSD with the following aims: (i) to elicit tonic immobility in humans in a laboratory setting, (ii) to identify an objective postural measure for this phenomenon, and (iii) to determine the cardiac correlates of tonic immobility.

\section{Methods and materials}

\subsection{Participants}

Thirty-three trauma-exposed subjects participated in the study. Eighteen patients with primary diagnoses of PTSD were recruited from an outpatient university clinic specialized in posttraumatic stress assessment and treatment. Psychiatric diagnoses were obtained using the Structured Clinical Interview for Diagnostic and Statistical Manual of Mental Disorders (DSM-IV) Axis I (First et al., 1997); translated and adapted to Portuguese by Del-Ben et al. (2001). Exclusion criteria were psychotic disorders, severe personality disorders, or significant cognitive impairment. Fifteen trauma-exposed (meeting criteria A1 and A2 of DSM-IV), age- and sex-matched participants without PTSD were also recruited. They scored $<35$ on the PTSD Checklist Civilian Version (PCL-C) (Berger et al., 2004; Weathers et al., 1993), one of the most conservative cutoffs for PTSD screening (McDonald and Calhoun, 2010). The predominant index trauma for both PTSD and non-PTSD participants was violence with weapons $(\mathrm{PTSD}=72 \%$; non-PTSD $=80 \%$ ). Patients were under pharmacological treatment with antidepressant drugs in adequate doses according to the recommended guidelines for PTSD (Friedman et al., 2008). Table 1 contains the characteristics of the participants.

The Ethics Review Board of the Institute of Psychiatry of the Federal University of Rio de Janeiro approved this study, and participants provided informed consent before assessment. Participants were informed that they could withdraw from the experiment at any time.

\subsection{Autobiographical scripts}

The protocol for symptom provocation was based on previous studies (Lang et al., 1980, 1983; Pitman et al., 1987). The participants meticulously narrated their worst traumatic event and described the physical reactions experienced during this event. The details about the traumatic events collected included date, time, location, sensory stimulus (e.g. smells, sounds, images, pain), behavioral reactions (e.g. fighting, paralyzing), emotional reactions (e.g. fear, anger), presence of others, etc. We summarized the individual scripts so that they ended at the most terrifying moment reported by the participants. Scripts comprised exactly 100 words each chronologically portraying the traumatic experience in the second person, present tense. The scripts were then presented to the participants in order to assure accuracy. Follow- ing this, a professional speaker recorded the 60-s scripts in a male, neutral voice for later playback.

\subsection{Data collection}

Two PC-compatible computers controlled script presentation and data acquisition of the postural and electrocardiographic parameters, running Presentation (Neurobehavioral Systems), Balance Clinic (AMTI's AccSway Plus Balance Platform) and Acknowledge (BIOPAC Systems Inc.) softwares.

\subsubsection{Posturography}

Body sway was estimated by measuring the displacement of the center of pressure (CoP) using a force platform (AccuswayPLUS, AMTI, USA). The posturographic signals were sampled at $50 \mathrm{~Hz}$ and low-pass filtered with a cutoff frequency at $5 \mathrm{~Hz}$. The area of postural sway was quantified by fitting the displacement of the CoP in the $x-y$ plane into an ellipse that encloses approximately $95 \%$ of the points of the CoP trajectory (Odenrick and Sandstedt, 1984; Prieto et al., 1992). The confidence ellipse was calculated using principal component analysis (Oliveira et al., 1996).

\subsubsection{Electrocardiography}

Electrocardiographic recordings were collected at a sampling frequency of $1000 \mathrm{~Hz}$ through an electrocardiograph ECG100C module coupled to the MP150 system (BIOPAC Systems Inc.). An off-line peak detection algorithm (derivative plus threshold) was used to estimate R-wave fiducial points, after which the series was screened by hand and corrected for artifacts. R-R intervals were averaged and converted to beats/min to express heart rates. A time-domain measure of heart rate variability was calculated by the root mean square of successive differences (RMSSD) between adjacent R-R intervals (Task Force of the European Society of Cardiology and the North American Society of Pacing and Electrophysiology, 1996).

\subsubsection{Psychometric assessment of immobility}

Immobility reports were obtained at two different moments: during the enrollment process (to retrospectively assess symptoms of immobility experienced during the actual traumatic event) and immediately after the autobiographical scriptdriven symptom provocation (to assess symptoms of immobility elicited by the trauma script). We used an adaptation (Lima et al., 2010; Rocha-Rego et al., 2009) of the four "motor" items from the Tonic Immobility Scale-Child form (TIS-C) (Forsyth et al., 2000). Each question was evaluated with a Likert scale varying from 0 to 6 Trained interviewers applied the scale at both moments and assured that participants had a clear understanding of each item. The adapted four "motor" items were as follows: (i) question: rate the degree to which you froze or felt paralyzed during the event, answer: $0=$ not at all frozen or paralyzed $/ 6=$ completely frozen or paralyzed; (ii) question: rate the degree to which you were unable to move even though not restrained during the event, answer: $0=$ could move freely $/ 6=$ could not move at all; (iii) question: rate the degree to which you were unable to call out or scream during the event even though you were willing to, answer: $0=$ felt able to scream $/ 6=$ wanted to scream but felt unable to; and (iv) question: rate the extent to which you felt unable to escape during the event even though you were willing to, answer: 0 = felt able to escape $/ 6$ = wanted to escape but remain fixed. Immobility report scores were calculated by adding the answer ratings for the four questions ( minimum $=0$; maximum $=24$ ).

\subsection{Procedure}

The experimental sessions occurred in a light- and sound-attenuated room with white walls. The force platform was placed at a distance of $50 \mathrm{~cm}$ from the fronta wall and equidistant to the lateral walls. A $10-\mathrm{cm}$ diameter cardboard circle was placed on the frontal wall at the height of the eyes of each participant. Participants had electrocardiogram electrodes attached and wore earphones (Vibration, Clone). To familiarize subjects with the experimental tools, participants were asked to stand for $120 \mathrm{~s}$ on the force platform with bare feet together and arms relaxed along the trunk for a training session. Then, they returned to the platform to start the testing session, comprised continuously of a 200 -s adaptation period (instructions: $60 \mathrm{~s}$; silent interval: $20 \mathrm{~s}$; neutral playback: $60 \mathrm{~s}$, silent interval: $60 \mathrm{~s}$ ), a 60 -s presentation of the autobiographical traumatic script, and, finally, a 60-s reactivity period. The analyses of biological variables were performed during the period succeeding the end of the audio-taped script presentation. Previous studies showed that postscript period was the most effective to reveal physiological responses (Lang et al., 1980, 1983; Pitman et al., 1987). In the present paradigm, the scripts were elaborated in a crescendo so that they ended at the most terrifying moment reported by the participants. This allowed for a similar level of exposure across participants at the post-script period. To assure an automatic reaction and avoid unwanted attentional interference in body sway (Pellecchia, 2003), the traumatic script was presented without the explicit instructions to "pay attention" and to "vividly try to re-experience" the situation.

The area of sway and mean heart rate were calculated during the 60-s period after the end of script presentation and the heart rate variability was calculated for a lesser duration $(40 \mathrm{~s})$ to discard arrhythmic beats presented by two patients. 


\subsection{Statistics}

The distribution of the variables used in the analyses was investigated by histograms and tests for normality. The best transformation was identified using the ladder command of Stata software, which searches a subset of the ladder of powers (Tukey, 1977) to obtain a normal distribution of the transformed variable.

\subsubsection{Psychometric variables}

We compared immobility scores reported during enrollment with those obtained after script-driven symptom provocation in laboratory. As no transformation was identified for normalizing, we used the Wilcoxon matched-pairs signed-ranks test. Interaction with type of participant (with and without PTSD) could not be formally tested with this non-parametric statistic.

\subsubsection{Biological variables}

2.5.2.1. Posturography. Our main goal was to investigate the impact of the immobility experienced (immobility scores) on an objective biological measure which could reflect this subjective experience. To accomplish this goal, a linear regression was fitted with the sway area as the dependent variable (biological measure) and immobility reported after symptom provocation as the independent variable (immobility experienced). This association was investigated controlling for type of participant (with and without PTSD). We also tested an interaction term between immobility report and type of participant. The area of sway was log-transformed to normalize its distribution.

2.5.2.2. Electrocardiography. To investigate the degree of relationship between cardiac parameters and immobility reports, we estimate the statistical correlations of immobility scores reported after symptom provocation with both heart rate and heart rate variability (RMSSD). RMSSD data was square root-transformed to normalize its distribution. No transformation was identified for normalizing heart rate distribution. Thus, we estimated the Pearson correlation coefficient for the RMSSD and the non-parametric Spearman correlation coefficient for the heart rate.

2.5.2.3. Complementary analysis. As a complementary analysis we used the Wilcoxon matched-pairs signed-ranks test to investigate the change of heart rate and heart rate variability and the change of area of sway from adaptation (neutral playback) to reactivity period among PTSD participants.

In all analyses, we considered $p$ values $\leq 0.05$ as statistically significant.

\section{Results}

\subsection{Immobility reports}

The instrument that estimated the degree of reported immobility revealed that script presentation in the laboratory evoked reliable immobility experiences. The range of the instrument covers from 0 (no immobility) to 24 (complete immobility). Seven participants scored at the upper quartile, that is, scores between 18 and 24 , meaning that they felt extreme immobility.

Furthermore, scores on reports related to script-driven symptom provocation did not differ from those obtained at enrollment $(Z=-1.05 ; p=0.29)$. This seemed to apply in particular for participants with PTSD. When data was investigated separately for type of participants, for those with PTSD the analysis revealed no difference $(Z=0.42, p=0.68$ ), whereas for those without PTSD the autobiographical script presentation evoked significantly lower scores in immobility report than those obtained during enrollment $(Z=-2.37, p=0.02)$.

\subsection{Biological measures}

\subsubsection{Body sway}

Regression analysis showed that higher reports of immobility were associated with lower amplitudes of body sway, as measured by the area of displacement during the 60-s period after presentation of the trauma script (beta $=-0.03, p=0.01$ ), controlling by type of participant. This indicates that for each additional point increment in the immobility score, the log of the area of sway decreased $0.03 \log \mathrm{mm}^{2}$. No interaction was found between immobility report and type of participant on the amplitude of body sway $(p=0.90)$.

\subsubsection{Heart rate and heart rate variability}

Due to a technical problem, ECG data was lost for one participant (with PTSD). Immobility scores on reports related to symptom provocation were positively correlated with heart rate ( $r h o=0.35$, $p=0.05$ ). For heart rate variability, there was an inverse correlation with immobility scores $(r=-0.39, p=0.03)$. In summary, the higher reports of immobility were correlated with accelerated heart rates and diminished heart rate variability.

\subsubsection{Illustrative data}

The essence of these findings is represented in Fig. 1, which shows the pooled raw data of postural and cardiac biomarkers in four of the participants with PTSD during the reactivity period. For two patients reporting high immobility (score 23 for case 1 ; score 24 for case 2), small sway areas parallel high heart rates, while for two other patients with low immobility (score 0 for case 3 ; score 3 for case 4), larger sway areas parallel lower heart rates. Also, heart rate variability was very low for the patients reporting high immobility (case 1, RMSSD: $3.5 \mathrm{~ms}$; case 2, RMSSD: $4.7 \mathrm{~ms}$ ) compared to the other two patients (case 3, RMSSD: $11.4 \mathrm{~ms}$; case 4, RMSSD: $16.7 \mathrm{~ms})$.

\subsubsection{Complementary analysis}

An additional analysis of the sample with PTSD corroborates the observations presented in Fig. 1. Scores above and below the mid value of 12 in the instrument that estimated the degree of reported immobility defined high and low immobility subgroups. The "high immobility subgroup" showed a significant increase in heart rate $(Z=-2.20, p=0.03)$, a significant decrease in heart rate variability $(Z=2.04, p=0.04)$, and no significant change in area of sway $(Z=-1.35, p=0.18)$ from adaptation to reactivity period. On the other hand, the "low immobility subgroup" significantly augmented the area of sway from adaptation to reactivity period $(Z=-2.31, p=0.02)$. No significant changes in heart rate $(Z=-1.16$, $p=0.25)$ and heart rate variability $(Z=0.60, p=0.55)$ were observed for this subgroup.

\section{Discussion}

This is the first study to induce a tonic immobility-like reaction in humans in the laboratory. Probing postural displacement, which reflects the dynamic activity of the motor systems, revealed a potential biomarker of this phenomenon. Reports of immobility driven by the trauma scripts predicted restriction of body sway. They also correlated with accelerated heart rates and with waning of heart rate variability.

\subsection{Posturography and the defense cascade}

Sensations of intense paralysis and inescapability after listening to the personal trauma scripts, as indicated by high scores on immobility questions, were linked with objective measures of body immobility. The present posturographic data can be compounded with other studies to add support for a human defense cascade. The intensity of perceived fear (Marx et al., 2008) appears to trigger (in a serial sequence) freeze, flight, fight and tonic immobility defensive strategies. "Freezing", characterized by reduced body sway in response to low threat, was measured in healthy volunteers during the viewing of aversive pictures (Azevedo et al., 2005; Facchinetti et al., 2006). Exposure to height challenges in healthy participants also reduced sway amplitude, possibly indicating a freezing reaction (Adkin et al., 2000; Carpenter et al., 2001). Interestingly, a recent study found that participants reporting "low fear" at elevated heights present reduced body sway as expected, but those experiencing "high fear" show enlarged, rather than reduced, body sway at increased heights (Davis et al., 2009). The latter results 

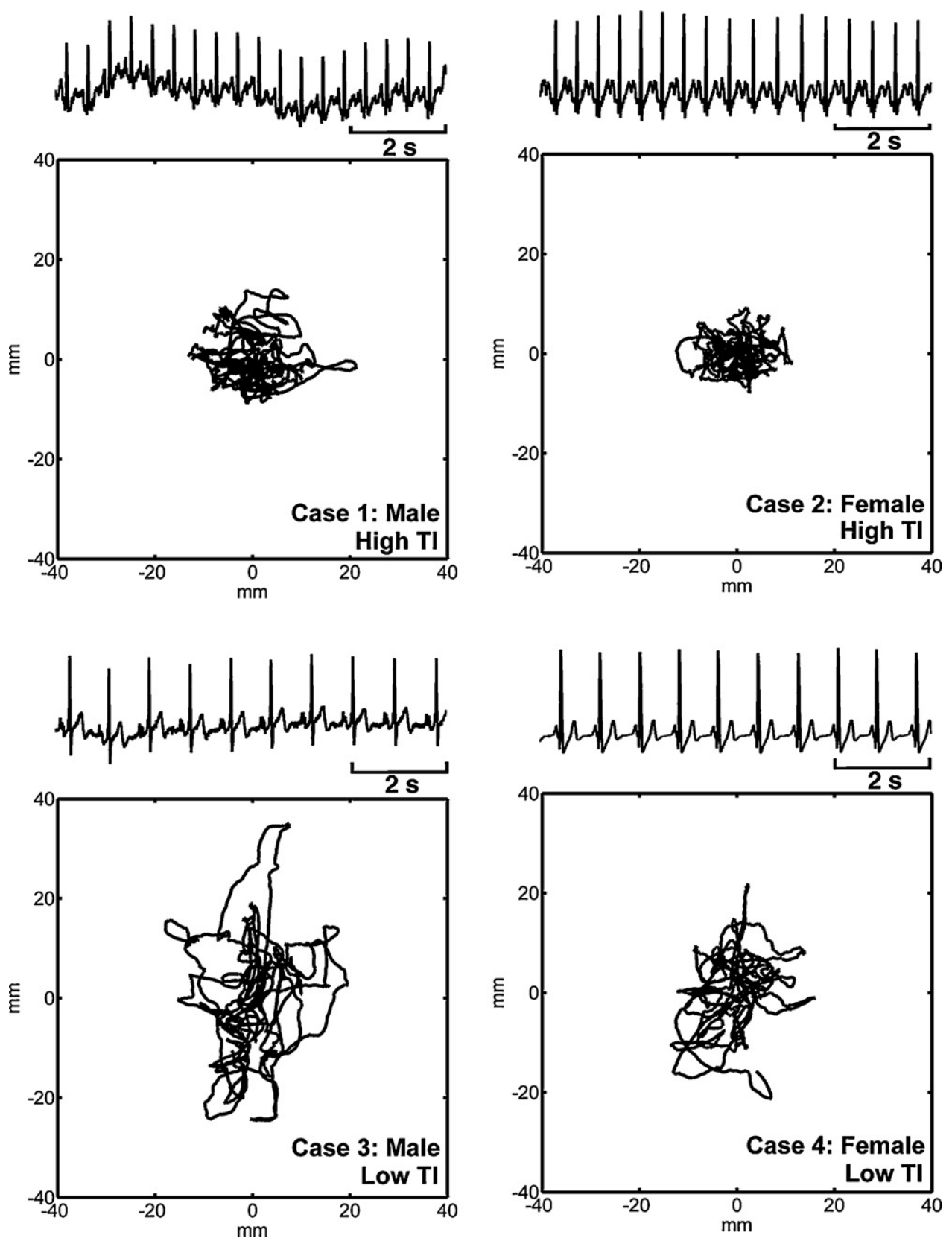

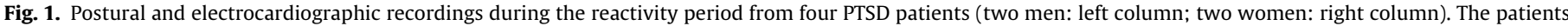

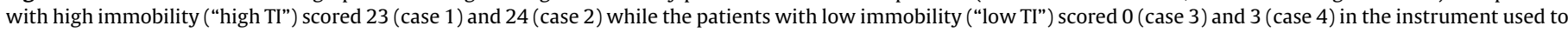

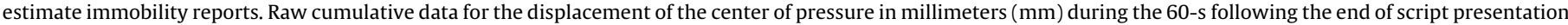
is depicted inside the squares for each patient. Eight-second samples of ECG recordings for each patient are presented above the respective body sway data.

from high-fear participants may be posturographic evidence of the "flight" stage. In the present study, we revealed reduced body sway in response to an overwhelming and inescapable perception of threat through posturographic recording in trauma-exposed participants, representing the tonic immobility stage of the defense cascade.

\subsection{Heart rate and tonic immobility}

The modulation of heart rate seems to differentiate two stages of immobility along the defense cascade. The freezing reaction to low threat contexts (e.g. pictures of mutilations) is accompanied by bradycardia (Azevedo et al., 2005; Facchinetti et al., 2006). On the other hand, the present study showed that tonic immobility-like reaction when reliving a traumatic experience is accompanied by tachycardia.

There are few reports of heart rate modulation during tonic immobility in vertebrates. Similar to our findings in humans, studies in rabbits (Carli, 1974), pigeons (Ratner, 1967) and quails (Valance et al., 2008) found tachycardia during tonic immobility, and the latter observed a reduction in heart rate variability. The aforementioned studies used a standardized procedure to induce tonic immobility in the laboratory, consisting of turning the animal on its back or side, holding it for a fixed time, and then releasing it and observing the tonic immobility state until the animal returns to its feet. Such maneuvers are an attempt to simulate the physical restraints that can result in the immobility of a prey when caught and/or carried by a predator. 
A study with wild rodents reported bradycardia and a high incidence of cardiac arrhythmias accompanying immobilization in a paradigm in which the recently captured animals were tested in an "open field" arena where they were subjected to sudden visual and auditory stimulation, as well as to a natural predator (Hofer, 1970). We previously showed that a freezing-like state can be induced in humans by a potential threat, and this reaction is accompanied by bradycardia (Azevedo et al., 2005; Facchinetti et al., 2006). However, though it is conceivable that the aforementioned work with wild rodents is indicative of a freezing state rather than tonic immobility, the reported observations do resemble tonic immobility in at least some of the tested animals (Hofer, 1970). The apparent controversy may result from a technical aspect, because in the section devoted to cardiac arrhythmias (pp. 641-642) the author mentioned that tachycardia may have been missed due to the very slow polygraph paper speed.

\subsection{Tonic immobility as an adaptive defensive trait}

The adaptive value is supported by the fact that the absence of movement increases the odds that a captured animal will escape, because a predator may loosen its grip if it assumes that the prey is indeed dead. For example, in ducks and pheasants, tonic immobility was shown to be of high survival value under predator attack (Rutting et al., 2007; Sargeant and Eberhardt, 1975). A report from informal interviews with trauma survivors (Ripley, 2008) also provides hints toward the adaptive value of tonic immobility in humans.

Susceptibility to tonic immobility was shown to vary across and within species (Gallup, 1974; Hennig, 1978; Ratner, 1967). The existence of different defensive traits within each species is widespread in the animal kingdom. Natural selection maintains a balance of these different defensive traits so that, under different environmental conditions, there are survivors (Korte et al., 2005). Presenting immobility in some contexts may increase the chances of survival, while in others; it may fail to be protective.

\subsection{PTSD and tonic immobility: clinical implications}

We found an association between increased immobility reports and decreased area of sway which holds independently of the presence/absence of PTSD. However, we also detected that the group of participants without PTSD scored significantly less on immobility reports driven by the trauma scripts compared to those obtained during enrollment. A substantial number of non-PTSD participants reported tonic immobility-like reaction during the actual trauma, but much fewer reported this reaction for the playback of their personal trauma. On the other hand, for the group of participants with PTSD, immobility scores after script-driven presentation were not statistically different from those obtained during enrollment. For PTSD patients, listening to their own trauma in the lab evoked reports of tonic immobility in a similar proportion of cases as in the actual experience. The presence of re-experiencing symptoms, a crucial criterion for PTSD diagnosis, may have played a key role in this context. This finding raises the possibility that in their daily life some PTSD patients may present tonic immobility-like reactions during the re-experiencing episodes.

It is worthy to note that participants with PTSD that experienced intense paralysis after script presentation underwent both a significant elevation of heart rate and a significant reduction of heart rate variability compared to previous levels. No change was observed in the area of body sway, which contrasted with the non-paralyzed patients who augmented the amplitude of sway but showed feeble modulation of cardiac parameters. Repeated activation of stress mediators can lead to dysregulation of allosta- sis on body and brain compromising stress resiliency and health (Kario et al., 2003; McEwen and Gianaros, 2010). In experimental animals, repeated inductions of tonic immobility potentiate this reaction (Ratner, 1967) and have hazardous long-term consequences, including death, in some cases (Liberson et al., 1961). It was previously shown that PTSD patients reporting peritraumatic tonic immobility were the most severely affected (Fiszman et al., 2008; Lima et al., 2010; Rocha-Rego et al., 2009). One could speculate that repeated episodes of tonic immobility may underpin the severity of the disorder in those patients, being largely unnoticed by clinicians and researchers.

PTSD patients who experienced higher levels of peritraumatic tonic immobility showed poor responses to pharmacotherapy with antidepressants (mainly selective serotonin reuptake inhibitors, SSRIs) (Fiszman et al., 2008; Lima et al., 2010), raising the need for more specific pharmacological agents. Systemic administration of the dopamine antagonist haloperidol was shown to reinforce some immobility reactions in some rodents (Fregoso-Aguilar et al., 2002; Meyer et al., 1984). Conversely, in humans, low-doses of amisulpride (which increase dopaminergic transmission) have been reported to reverse motor conversion paralysis in a case resistant to an SSRI at an adequate dose (Oulis et al., 2009). These findings may draw the attention of researchers and clinicians to a neglected class of medications in the treatment of SSRI-resistant PTSD (see Berger et al., 2009), namely dopamine activity enhancers. Further elucidation of the neurochemical networks involved in tonic immobility may also aid the development of future preventive and therapeutic strategies to prepare and heal people confronted with traumatic events.

\subsection{Humans do present tonic immobility: benefits of knowledge}

Experiencing tonic immobility may elicit the horror of being locked-in. Two patients in the present sample reported the urge to move and the terror of being trapped in their immobile body during a shooting incident as their index trauma. One of them additionally felt painfully guilty and ashamed. She was a teacher in a primary school, and finding herself involuntary immobilized prevented her from acting to protect her students.

Rape victims who present tonic immobility during the sexual assault are frequently overwhelmed by shame and can receive less empathy, as well as less legal protection, because successful prosecution of sexual offenders in legal courts is strongly related to the signs of resistance in most countries (Freyd, 2008; Fuse et al., 2007; Heidt et al., 2005). Professionals, like police officers and the military, who face traumatic events as part of their jobs, can suffer administrative restrictions if they paralyze in duty.

Tonic immobility still remains largely unrecognized in humans as an automatic and uncontrollable reaction under extreme life threatening circumstances. Thus, essential steps to alleviate entrapment symptoms, guilt and prejudice in the aftermath of tonic immobility are the recognition of tonic immobility and dissemination of this knowledge to the public.

\section{Conclusion}

We succeeded in experimentally inducing tonic immobility in humans and recording its biological correlates, indicating that tonic immobility is preserved in humans as an involuntary defensive strategy to life-threatening events. Restricted body sway, in parallel with an accelerated heart rate (and attenuated heart rate variability), under trauma cues are biological markers of tonic immobility, a fright reaction for which cumulative clinical evidence has linked to the severity of the most disruptive sequelae of trauma exposure - PTSD. 


\section{Financial disclosure}

The authors report no biomedical financial interests or potential conflicts of interest.

\section{Acknowledgements}

We extend special thanks to Sonia Gleiser for her managing assistance and to the following individuals for their assistance in data collection: Alessandra Lima, Alessandra Teixeira, Ana Mendonça-de-Souza, and Mariana Luz. We also thank the anonymous reviewers for their helpful comments. This work was supported by National Council for Scientific and Technological Development (CNPq) and Carlos Chagas Filho Foundation of Research Support in Rio de Janeiro (FAPERJ). Partial support was provided by FINEP, CAPES and CNC.

\section{References}

Abrams, M.P., Carleton, R.N., Taylor, S., Asmundson, G.J.G., 2009. Human tonic immobility: measurement and correlates. Depression and Anxiety 26, 550-556.

Adkin, A.L., Frank, J.S., Carpenter, M.G., Peysar, G.W., 2000. Postural control is scaled to level of postural threat. Gait and Posture 12, 87-93.

Azevedo, T.M., Volchan, E., Imbiriba, L.A., Rodrigues, E.C., Oliveira, J.M., Oliveira, L.F. et al., 2005. A freezing-like posture to pictures of mutilation. Psychophysiology $42,255-260$.

Bados, A., Toribio, L., Garcia-Grau, E., 2008. Traumatic events and tonic immobility. The Spanish Journal of Psychology 11, 516-521.

Berger, W., Mendlowicz, M.V., Marques-Portella, C., Kinrys, G., Fontenelle, L.F., Marmar, C.R., et al., 2009. Pharmacologic alternatives to antidepressants in posttraumatic stress disorder: a systematic review. Progress in Neuropsychopharmacology and Biological Psychiatry 33, 169-180.

Berger, W., Mendlowicz, M.V., Souza, W., Figueira, I., 2004. Semantic equivalence of the Portuguese version of the Post-Traumatic Stress Disorder Checklist-Civilian Version (PCL-C) for the screening of post-traumatic stress disorder. Revista de Psiquiatria do Rio Grande do Sul 26, 167-175

Bovin, M.J., Jager-Hyman, S., Gold, S.D., Marx, B.P., Sloan, D.M., 2008. Tonic immobility mediates the influence of peritraumatic fear and perceived inescapability on posttraumatic stress symptom severity among sexual assault survivors. Journal of Traumatic Stress 21, 402-409.

Bradley, M.M., Codispoti, M., Cuthbert, B.N., Lang PJ., 2001. Emotion and motivation. I. Defensive and appetitive reactions in picture processing. Emotion 1, 276-298.

Carli, G., 1974. Blood pressure and heart rate in the rabbit during animal hypnosis. Electroencephalography and Clinical Neurophysiology 37, 231-237.

Carpenter, M.G., Frank, J.S., Silcher, C.P., Peysar, G.W., 2001. The influence of postural threat on the control of upright stance. Experimental Brain Research 138, 210-218.

Coutinho, M.R., Menescal-De-Oliveira, L., 2010. Role of homocysteic acid in the guinea pig (Cavia porcellus) anterior cingulate cortex in tonic immobility and the influence of NMDA receptors on the dorsal PAG. Behavioural Brain Research 208, 237-242.

Davis, J.R., Campbell, A.D., Adkin, A.L., Carpenter, M.G., 2009. The relationship between fear of falling and human postural control. Gait and Posture 29, 275-279.

Del-Ben, C.M., Vilela, J.A., Crippa, J.A., Hallak, J.C., Labate, C.M., Zuardi, A.W., 2001. Confiabilidade da "Entrevista Clínica Estruturada para DSM-IV - Versão Clínica" traduzida para o português. Revista Brasileira de Psiquiatria 23, 156-159.

Facchinetti, L.D., Imbiriba, L.A., Azevedo, T.M., Vargas, C.D., Volchan, E., 2006. Postural modulation induced by pictures depicting prosocial or dangerous contexts. Neuroscience Letters 410, 52-56.

Ferreira, M.D., Menescal-De-Oliveira, L., 2009. Role of dorsal raphe nucleus 5-HT(1A) and $5-\mathrm{HT}(2)$ receptors in tonic immobility modulation in guinea pigs. Brain Research 1285, 69-76.

First, M.B., Spitzer, R.L., Gibbon, M., Williams, J.B.W., 1997. Structured Clinical Interview for DSM-IV Axis I Disorders (SCID I), Clinical Version. American Psychiatric Press, Inc., Washington, DC.

Fiszman, A., Mendlowicz, M.V., Marques-Portella, C., Volchan, E., Coutinho, E.S., Souza, W.F., et al., 2008. Peritraumatic tonic immobility predicts a poor response to pharmacological treatment in victims of urban violence with PTSD. Journal of Affective Disorders 107, 193-197.

Forsyth, J.P., Marx, B., Fusé, T.M.K., Heidt, J., Gallup, G.G., 2000. The Tonic Immobility Scale. Child Form. Authors, Albany, NY.

Fregoso-Aguilar, T., Uriostegui, T., Zamudio, S., De la, C.F., 2002. The differential effect of haloperidol and repetitive induction on four immobility responses in mouse and guinea pig. Behavioural Pharmacology 13, 253-260.

Freyd, J.J., 2008. What juries don't know: dissemination of research on victim response is essential for Justice. Trauma Psychology Newsletter 3, 15-18.

Friedman, M.J., Davidson, J.R., Stein, D.J., 2008. Effective treatments for PTSD: practice guidelines from the International Society for Traumatic Stress Studies. In:
Foa, E.B., Keane, T.M., Friedman, M.J., Chen, J.A. (Eds.), Psychopharmacology for Adults., second ed. The Guilford Press, New York, pp. 245-268.

Fuse, T., Forsyth, J.P., Marx, B., Gallup, G.G., Weaver, S., 2007. Factor structure of the Tonic Immobility Scale in female sexual assault survivors: an exploratory and Confirmatory Factor Analysis. Journal of Anxiety Disorders 21, 265-283.

Galliano, G., Noble, L.M., Travis, L.A., Puechl, C., 1993. Victim reactions during rape sexual assault - a preliminary-study of the immobility response and its correlates. Journal of Interpersonal Violence 8, 109-114.

Gallup Jr., G.G., 1974. Animal hypnosis: factual status of a fictional concept. Psychological Bulletin 81, 836-853.

Heidt, J.M., Marx, B.P., Forsyth, J.P., 2005. Tonic immobility and childhood sexua abuse: a preliminary report evaluating the sequela of rape-induced paralysis. Behaviour Research and Therapy 43, 1157-1171.

Hennig, C.W., 1978. Tonic immobility in the squirrel monkey (Saimiri sciureus). Primates 19, 342.

Hofer, M.A., 1970. Cardiac and respiratory function during sudden prolonged immobility in wild rodents. Psychosomatic Medicine 32, 633-647.

Humphreys, K.L., Sauder, C.L., Martin, E.K., Marx, B.P., 2010. Tonic immobility in childhood sexual abuse survivors and its relationship to posttraumatic stress symptomatology. Journal of Interpersonal Violence 25, 358-373.

Kario, K., McEwen, B.S., Pickering, T.G., 2003. Disasters and the heart: a review of the effects of earthquake-induced stress on cardiovascular disease. Hypertension Research 26, 355-367.

Korte, S.M., Koolhaas, J.M., Wingfield, J.C., McEwen, B.S., 2005. The Darwinian concept of stress: benefits of allostasis and costs of allostatic load and the trade-offs in health and disease. Neuroscience and Biobehavioral Reviews 29, 3-38.

Lang, P., Bradley, M.M., Cuthbert, B.N., 1997. Motivated attention: affect, activation and, action. In: Lang, P.J., Simons, R.F., Balaban, M.T. (Eds.), Attention and Orienting: Sensory and Motivational Processes. Lawrence Erlbaum Associates, New Jersey, pp. 97-135.

Lang, P.J., Kozak, M.J., Miller, G.A., Levin, D.N., McLean Jr., A., 1980. Emotiona imagery: conceptual structure and pattern of somato-visceral response. Psychophysiology $17,179-192$.

Lang, P.J., Levin, D.N., Miller, G.A., Kozak, M.J., 1983. Fear behavior, fear imagery, and the psychophysiology of emotion: the problem of affective response integration. Journal of Abnormal Psychology 92, 276-306.

Leite-Panissi, C.R., Coimbra, N.C., Menescal-De-Oliveira, L., 2003. The cholinergic stimulation of the central amygdala modifying the tonic immobility response and antinociception in guinea pigs depends on the ventrolateral periaqueducta gray. Brain Research Bulletin 60, 167-178.

Leite-Panissi, C.R., Ferrarese, A.A., Terzian, A.L., Menescal-De-Oliveira, L., 2006. Serotoninergic activation of the basolateral amygdala and modulation of tonic immobility in guinea pig. Brain Research Bulletin 69, 356-364.

Liberson, W.T., Smith, R.W., Stern, A., 1961. Experimental studies of the prolonged "hypnotic withdrawal" in guinea pigs. Journal of Neuropsychiatry 3, 28-34

Lima, A.A., Fiszman, A., Marques-Portella, C., Mendlowicz, M.V., Coutinho, E.S. Maia, D.C., et al., 2010. The impact of tonic immobility reaction on the prognosis of posttraumatic stress disorder. Journal of Psychiatry Research 44 224-228

Marks, I.M., 1987. Fears, Phobias and Rituals. Oxford University Press, New York.

Marx, B.P., Forsyth, J.P., Gallup, G.G., Fusé, T., Lexington, J.M., 2008. Tonic immobility as an evolved predator defense: implications for sexual assault survivors. Clinica Psychology: Science and Practice 15, 74-90.

Maser, J.D., Gallup, G.G., 1977. Tonic immobility and related phenomena: a partially annotated, tricentennial bibliography, 1636-1976. Psychological Record 27, 177-217.

McDonald, S.D., Calhoun, P.S., 2010. The diagnostic accuracy of the PTSD Checklist: a critical review. Clinical Psychology Review 30, 976-987.

McEwen, B.S., Gianaros, P.J., 2010. Central role of the brain in stress and adaptation: links to socioeconomic status, health, and disease. Annals of the New York Academy of Sciences 1186, 190-222.

Meyer, M.E., Smith, R.L., Van, H.C., 1984. Haloperidol differentially potentiates tonic immobility, the dorsal immobility response, and catalepsy in the developing rat. Developmental Psychobiology 17, 383-389.

Mobbs, D., Marchant, J.L., Hassabis, D., Seymour, B., Tan, G., Gray, M., et al., 2009 From threat to fear: the neural organization of defensive fear systems in humans. Journal of Neuroscience 29, 12236-12243.

Monassi, C.R., Menescal-De-Oliveira, L., 2004. Serotonin 5-HT2 and 5-HT1A receptors in the periaqueductal gray matter differentially modulate tonic immobility in guinea pig. Brain Research 1009, 169-180.

Odenrick, P., Sandstedt, P., 1984. Development of postural sway in the normal child Human Neurobiology 3, 241-244.

Oliveira, L., Hoffmann, A., Menescal-De-Oliveira, L., 1997. The lateral hypothalamus in the modulation of tonic immobility in guinea pigs. Neuroreport 8,3489 3493

Oliveira, L.F., Simpson, D.M., Nadal, J., 1996. Calculation of area of stabilometric signals using principal component analysis. Physiological Measurement 17, 305-312.

Oulis, P., Kokras, N., Papadimitriou, G.N., Masdrakis, V.G., 2009. Adjunctive lowdose amisulpride in motor conversion disorder. Clinical Neuropharmacology 32, 342-343.

Pellecchia, G.L., 2003. Postural sway increases with attentional demands of concurrent cognitive task. Gait and Posture 18, 29-34.

Pitman, R.K., Orr, S.P., Forgue, D.F., de Jong, J.B., Claiborn, J.M., 1987. Psychophysiologic assessment of posttraumatic stress disorder imagery in Vietnam combat veterans. Archives of General Psychiatry 44, 970-975. 
Prieto, T.E., Myklebust, J.B., Myklebust, B.M., 1992. Postural steadiness and ankle compliance in the elderly. IEEE Engineering in Medicine and Biology 11, 25-27.

Ratner, S.C., 1967. Comparative aspects of hypnosis. In: Gordon, J.E. (Ed.), Handbook of Clinical and Experimental Hypnosis. Macmillan, New York, pp. 550-587.

Ripley, A., 2008. Paralysis: playing dead in French class. In: The Unthinkable: Who Survives When Disaster Strikes and Why. Three Rivers Press, New York, pp. $163-178$

Rocha-Rego, V., Fiszman, A., Portugal, L.C., Garcia, P.M., de, O.L., Mendlowicz, M.V., et al., 2009. Is tonic immobility the core sign among conventional peritraumatic signs and symptoms listed for PTSD? Journal of Affective Disorders 115, 269-273.

Rutting, T., Brandt, H., Claubb, W., Dzapo, V., Selzer, D., 2007. Comparative study of predator avoidance behaviour of pheasants (Phasianus colchicus) of different genetic origin. European Journal of Wildlife Research 53, 171-177.

Sargeant, A.B., Eberhardt, L.E., 1975. Death feigning by ducks in response to predation by red foxes (Vulpes fulva). American Midland Naturalist 94, 108-119.
Task Force of the European Society of Cardiology and the North American Society of Pacing and Electrophysiology, 1996. Heart rate variability: standards of measurement, physiological interpretation, and clinical use. Circulation 93 , 1043-1065.

Tukey, J.W., 1977. Exploratory Data Analysis. Reading, MA.

Valance, D., Despres, G., Richard, S., Constantin, P., Mignon-Grasteau, S., Leman, S. et al., 2008. Changes in heart rate variability during a tonic immobility test in quail. Physiology and Behavior 93, 512-520.

Vieira, E.B., Menescal-De-Oliveira, L., Leite-Panissi, C.R., 2011. Functional mapping of the periaqueductal gray matter involved in organizing tonic immobility behavior in guinea pigs. Behavioral Brain Research 216, 94-99.

Weathers, F.W., Litz, B.T., Herman, D.S., Huska, J.A., Keane, T.M., 1993. The PTSD Checklist (PCL): reliability, validity, and diagnostic utility. In: Paper presented at the Annual Meeting of International Society for Traumatic Stress Studies, San Antonio, TX 